Stefan Kubica, Hagen Ringshausen, Jörg Reiff-Stephan, Marius Schlingelhoff (Hrsg.) 1. Automobil Symposium Wildau: Tagungsband Technische Hochschule Wildau 2016
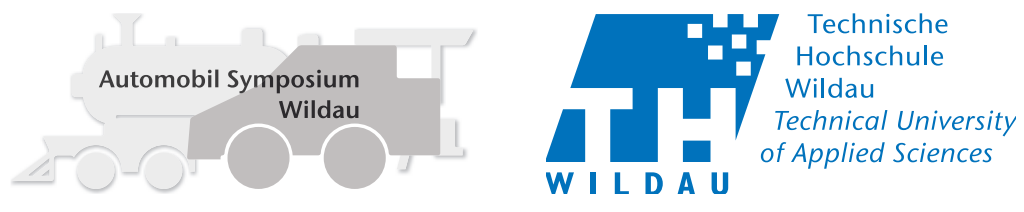

\title{
Data-Mining-Methoden zur frühzeitigen Erkennung bedrohlicher Situationen im Fahrzeug
}

\section{Einleitung}

Das autonome Fahren ist eines der großen Themen im Automobilbereich der Gegenwart. Umsatzstarke Software-Unternehmen gehen Kooperationen mit Unternehmen der Automobilbranche ein, um das automatisierte Fahren schnellstmöglich voranzutreiben (Fuest 2015). Gleichzeitig forschen diverse Universitäten an dem Thema und zeigen bereits weitgehende Fortschritte. Exemplarisch ist die Universität Ulm zu nennen, die bereits Testfahrten im urbanen Gebiet erfolgreich absolviert hat (Dietmayer 2014). Auch die Politik fördert den Forschungsschwerpunkt zunehmend. Als Beispiel ist das Förderprogramm »Elektroniksysteme für das vollautomatisierte Fahren (ELEVATE)« zu nennen, welches vom Bundesministerium für Bildung und Forschung angeboten wird (Bundesministerium für Bildung und Forschung 2015). Diese intensive Forschung, die gleichzeitig von der Wirtschaft, der Wissenschaft und der Politik vorangetrieben wird, zeigt wie viel Potential das Thema besitzt. Auch dadurch stellen sich neue Herausforderungen an die Sensorik innerhalb der Fahrzeuge. Aktuell wird der Großteil der Sensordaten einmalig für den jeweiligen Zweck genutzt und anschließend verworfen.

An diesen Punkt setzen das Vorhaben und der damit verbundene Einsatz von Data-Mining-Methoden an. Ziel ist die systematische Auswertung von Daten im Fahrzeug und von außerhalb (Car2X) in Echtzeit, um aktuelle Fahrsituationen mit einem digitalen Gedächtnis abzugleichen und somit einen kurzen Blick in die Zukunft abzuleiten. Hierbei werden Situationen durch Muster erkannt und Fahrzeug bzw. Fahrer konditioniert, um die Situation positiv zu beeinflussen.

Das Vorhaben ist nicht ausschließlich auf das autonome Fahren ausgerichtet, sondern auch als Hilfe für fahrergelenkte Fahrzeuge konzipiert.

\section{Nutzen von Data-Mining-Methoden im Fahrzeug}

Um Messwerte zu erhalten, ist in einem modernen Fahrzeug eine Vielzahl von Sensoren verbaut. Die erfassten Messdaten dieser Sensoren werden durch die verbauten Steuergeräte verwertet und falls notwendig werden Aktoren genutzt, um auf die Werte zu reagieren. Dabei werden die meisten Sensordaten nach dieser Nutzung gelöscht und nur die aktuellen Messwerte verwendet. Dies bietet ein Potential für eine Nutzung. Ein Erkenntnisgewinn aufgrund einer Nachverfolgung der Daten ist ermöglicht und durch eine Historisierung dieser können mithilfe von Data-Mining-Methoden Muster selektiert werden, die relevante Situationen wiedererkennbar machen.

Die möglichen relevanten Situationen sind vielfältig und weit gefächert. Exemplarisch dienen die folgenden zwei Beispiele für das Verständnis möglicher Situationen.

Die Erkennung von Fahrzuständen, wie die Fahrt auf der Autobahn ist ein Beispiel für das Nutzen von Data-Mining-Methoden auf Fahrzeugdaten. Es gibt verschiedene Ansätze einer Lösung dieser Erkennung. Durch die Positionsbestimmung mithilfe der Satellitenortung kann anhand der Position leicht festgestellt werden, dass sich das Fahrzeug auf der Autobahn befindet. Antennen für die Satellitensysteme GPS, Galileo oder GLONASS etc. sind in einigen Fahrzeugen verbaut, da sie für integrierte $\mathrm{Na}$ vigationssysteme verwendet werden. Wie kann aber eine Feststellung realisiert werden, wenn kein solches System verbaut ist? Eine weitere Option der Fahrzustandsermittlung »Autobahnfahrt« ist die Bestimmung der Geschwindigkeit. Auf Landstraßen kann die Geschwindigkeit aber ebenso $100 \mathrm{~km} / \mathrm{h}$ betragen und bei der Autobahnfahrt liegt sie bedingt durch stockenden Verkehr oder Baustellen oftmals auch unterhalb dieses Wertes. Folglich ist damit keine eindeutige Bestimmung möglich. Hier setzt die Mustererkennung an, indem sie die Lenkradwinkelsensordaten auswertet und wenn diese über einen bestimmten Zeitraum im vorgegeben Bereich sind, kann auf eine Autobahnauf- oder Abfahrt geschlossen werden. Diese Auf- sowie Abfahrten haben festgeschriebene Winkel und Radien. Daher kann durch die Data-Mining-Methoden auf Basis der historisierten Fahrzeugdaten eine eindeutige Bestimmung durchgeführt werden. Die Fahrzustandsermittlung »Autobahnfahrt« ist nicht zeitkritisch und aus diesem Grund ist keine Echtzeit notwendig. Wenn das Ergebnis wenige Sekunden später vorhanden ist, ist es für den weiteren Fahrprozess nicht hinderlich. Ein Beispiel für eine zeitkritische Anwendung ist die Erkennung eines unausweichlichen Verkehrsunfalls. Eine Mustererkennung auf Basis aufgenommener Unfälle (vor, während und nach dem Unfall) ermöglicht eine Voraussicht des Systems um einen kurzen Augenblick 
in die Zukunft. Selbst ein Ausblick im Millisekundenbereich kann über die schwere des Verkehrsunfalls und die damit verbundenen Verletzungen sowie Schäden am Fahrzeug entscheidend sein. Die Konditionierung des Fahrzeuges kurz vor Eintritt einer solchen Situation kann die entscheidenden Nuancen für die resultierende Schadensschwere bedeuten. Beispielsweise können das rechtzeitige Straffen des Gurtes, das exakt getimte Auslösen der Airbags, die in die optimale Höhe gefahrene Kopfstütze und eine automatische Vollbremsung Teil dieser Fahrzeugkonditionierung sein. Diese Reaktionen auf den bevorstehenden Unfall sind wichtig für die Gesundheit der Insassen und können im Extremfall über Leben und Tod dieser Menschen entscheiden. in den Bereichen Unfallprädiktion, autonomes Fahren sowie Sensorfusion angewandt werden. Das Potential des Forschungsgebiets ist vorhanden und in Zukunft kann es ausgeschöpft werden.

Aus der Vereinigung von Fahrzeugdaten und Business Intelligence entsteht dadurch »Vehicle Data Intelligence«. Die Wortschöpfung beschreibt die Bestandteile und die Idee des Vorhabens.

\section{Vorhabenbeschreibung}

Ziel des Vorhabens ist ein Echtzeit-Data-Mining von Fahrzeugdaten. Zu diesem Zweck entsteht ein Realtime-Data-Warehouse, welches zur Bereitstellung der

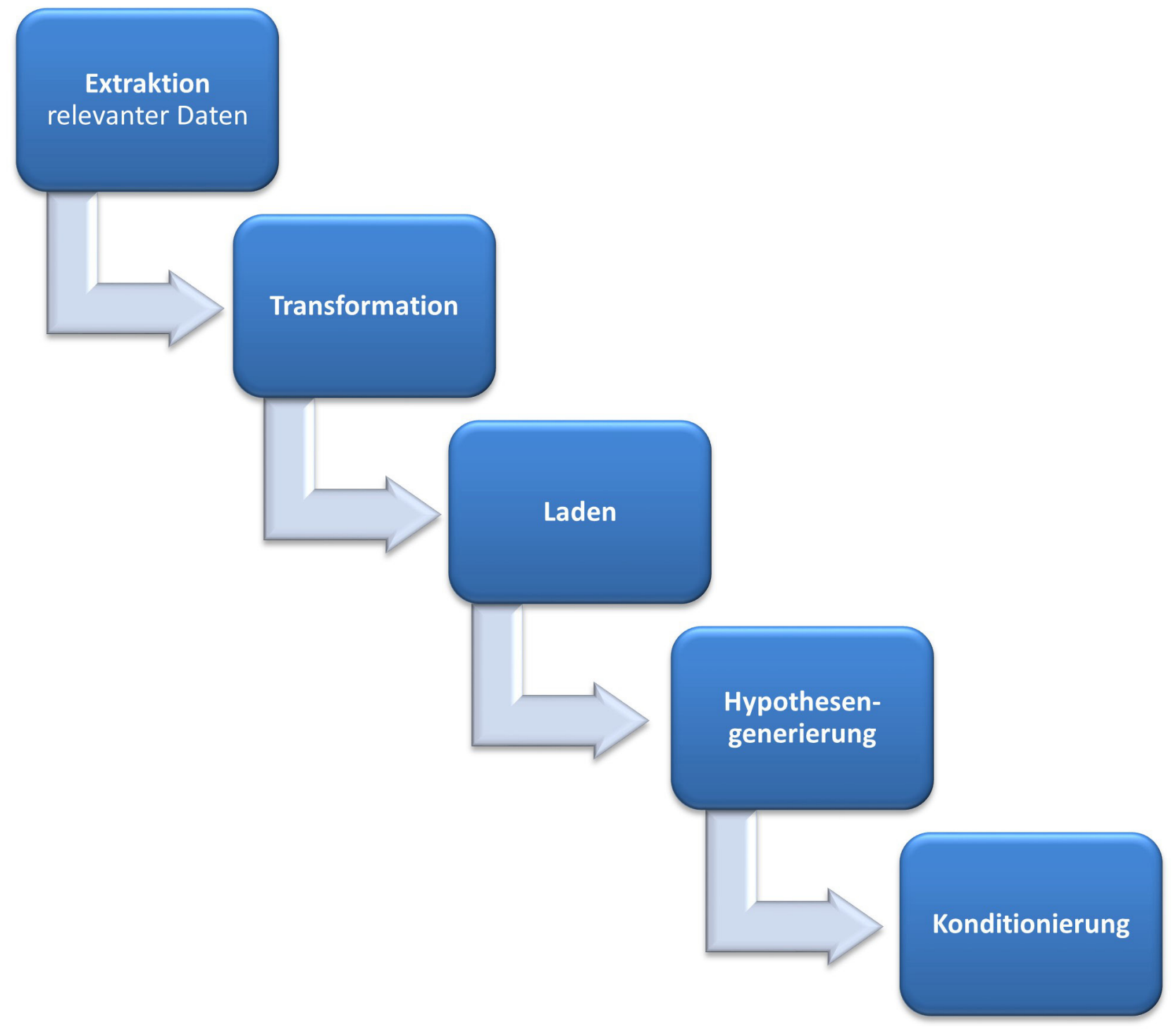

Abb. 1 Schrittweise Vorgehensweise des Verfahrens (Kubica 2015: 13)

Die beiden genannten Beispiele zeigen exemplarisch, wie weit gefächert die Anwendungen von Data-Mining-Methoden und die Historisierung von Fahrzeugdaten sind. Der Einsatz dient sowohl Comfort- wie auch Safety-Funktionen im Fahrzeug und die Nutzung kann analysierbarer Daten dient. Für die Verwertung der Fahrzeugdaten werden Data-Mining-Methoden angewandt. Diese Datenverwertung gliedert sich in Teilschritte von der Datengewinnung bis zur Konditionierung des Fah- 
rers bzw. des Fahrzeuges. Die Abb. 1 zeigt die grundlegenden Teilschritte als Übersicht.

Abb. 1 zeigt als ersten Schritt die Extraktion der relevanten Daten. Prof. Dr.-Ing. Christian Wietfield schätzt die permanenten Daten, die ausschließlich über den CAN-Bus täglich gesendet werden auf 12 GByte (Nestler 2015). Es wird deutlich, dass hohe Datenmengen im Fahrzeug entstehen. Eine Auswertung der gesamten Datenmenge ist möglich, würde aber den Gesamtprozess verlangsamen. Gerade bei zeitkritischen Anwendungsfällen ist dies kontraproduktiv und daher müssen die Daten anhand ihrer Relevanz gefiltert werden. Dadurch wird das Datenvolumen signifikant verringert und die Auswertung der Daten beschleunigt.

Nach der Extraktion der Daten folgt im nächsten Prozessschritt die Transformation dieser. Durch die Transformation der Daten wird sichergestellt, dass diese gleichgeschaltet und zeitlich synchronisiert sind. Es entsteht eine gemeinsame Datenbasis, die eine Weiterverarbeitung der Daten ermöglicht.

Die gefilterten und transformierten Fahrzeugdaten werden im dritten Schritt dem Echtzeitsystem hinzugefügt. Mittels dieser Maßnahme sind die Echtzeitdaten historisiert und die Tracing-Datensätze auswertbar.

Die Generierung von Hypothesen ist der Folgeschritt der Vorgehensweise des Verfahrens. In Folge dessen wird die
Auswertung der historisierten Datensätze ermöglicht. Der Fokus liegt auf der Erkennung vorhandener Muster. Die bereits bekannten Muster werden mit den historisierten Daten verglichen und auf eine Übereinstimmung überprüft. Wenn eine Übereinstimmung festgestellt wird (Matching), werden Wahrscheinlichkeitsberechnungen durchgeführt. Dabei wird ermittelt, mit welcher Wahrscheinlichkeit eine Situation vorliegt. Neben den bekannten Mustern wird das System über ein digitales Gedächtnis verfügen und selbstständig Muster ergänzen, ändern oder verwerfen. Das digitale Gedächtnis wird zudem die Wahrscheinlichkeiten für eintreffende Situationen historisieren und somit die Vorhersage konkretisieren. Die Historisierung der Wahrscheinlichkeiten führt zu Präzisierungen der Vorhersagen und somit zu einer genaueren Ermittlung von relevanten Situationen. Wird eine Situation von Bedeutung selektiert, folgt im nächsten Schritt ggf. eine Konditionierung von Fahrzeug und/oder des Fahrers. Ansonsten wiederholen sich die Schritte eins bis vier in einer Schleife.

Bei einer hohen Wahrscheinlichkeit eines Eintritts einer relevanten Situation muss je nach Situation prädiktiv eingegriffen werden. Wie die Prädiktion durchgeführt wird, ist im System hinterlegt. Dabei wird entweder das Fahrzeug, der Fahrer oder beides konditioniert. Eine Konditionierung des Fahrers wäre exemplarisch der Ein-

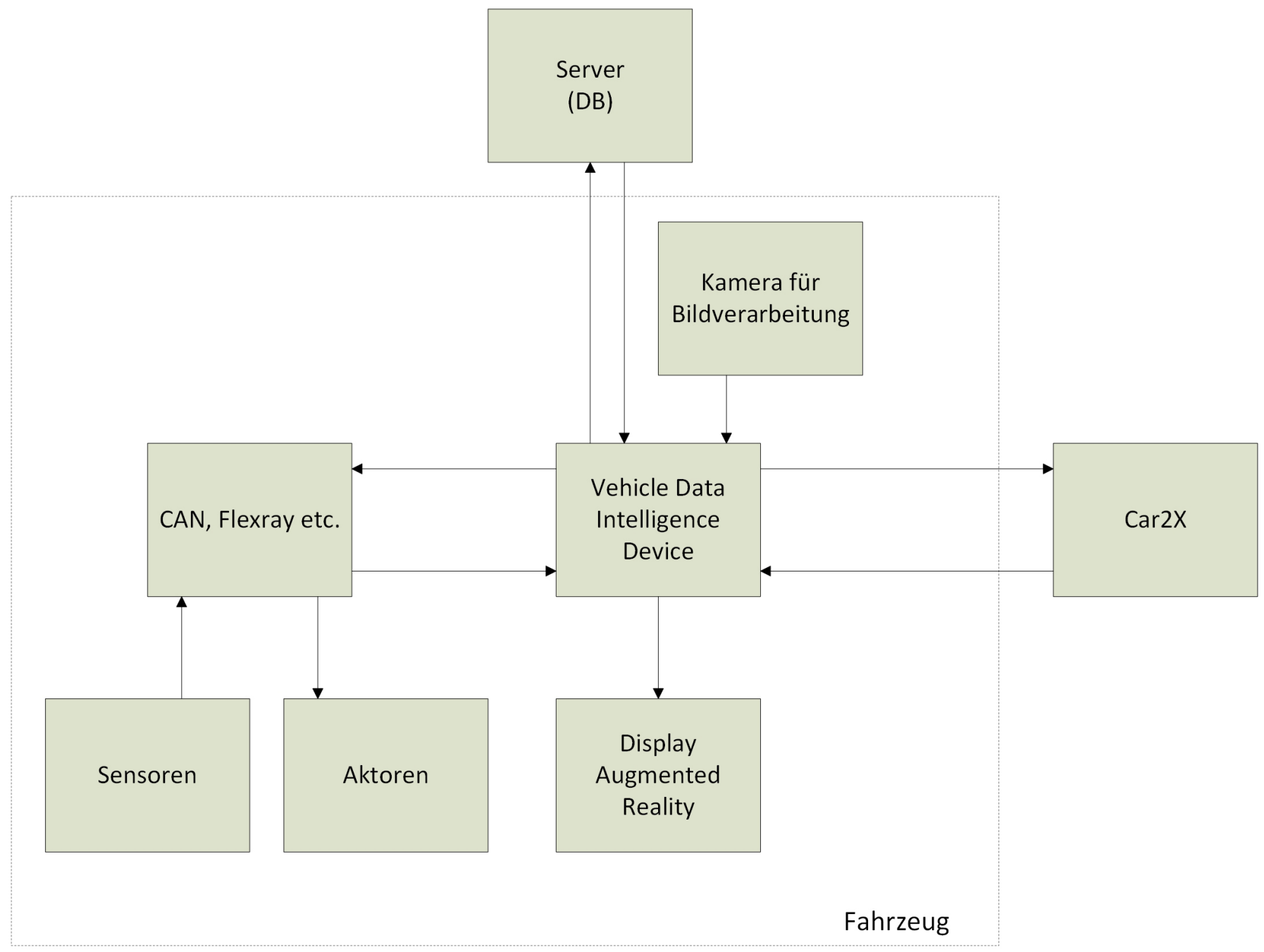

Abb. 2 Systemintegration und -Aufbau des Vehicle Data Intelligence Devices 
satz von optischen, akustischen oder haptischen Mitteln als Warnung für diesen. Für die Fahrzeugkonditionierung werden Aktoren von den eingebauten Steuergeräten verwendet.

Das Vorhaben zielt in der ersten Phase auf eine fahrzeuginterne Verwendung ab. Das heißt, es werden die verbauten Sensoren, Steuergeräte und Aktoren verwendet. Kommunikation zu anderen Fahrzeugen und der Infrastruktur ist zu einer späteren Phase angedacht. Die Car2X-Kommunikation, beispielsweise im 5,9 GHzBand nach IEEE 802.11p-Standard (IEEE Computer Society 2010), bietet weiteres Potential für das Vorhaben. Es werden durch die Kommunikation weitere Daten erhalten, die im Realtime-Data-Warehouse verwertet werden. Es entstehen neue Muster, welche in der Auswertung selektiert werden können. Folglich werden potentielle Situationen genauer vorhergesagt und das Erkennen wird wahrscheinlicher.

Ein weiterer Vorteil der Car2X-Kommunikation ist die Übertragung erlernter Muster des digitalen Gedächtnisses an andere Teilnehmer. Durch das Teilen der Muster wächst die Wahrscheinlichkeit des Erkennens relevanter Situationen und zudem wird das selbstständige Lernen im »Schwarm« unterstützt.

\section{Konzept Vehicle Data Intelligence}

Die Forschung und Entwicklung im Bereich Vehicle Data Intelligence ist aktuell konzeptionell und theoretisch.

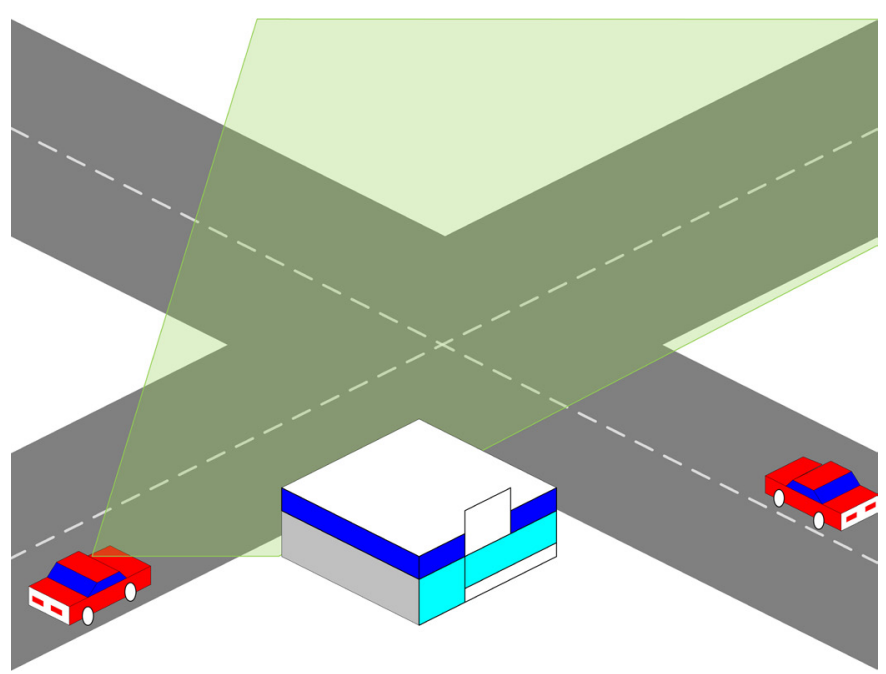

Abb. 3 Schematische Darstellung des Sichtbereichs des Fahrers vor einer Verkehrskreuzung

Die Ergebnisse sind bisher auf die Voruntersuchungen der Möglichkeiten einer solchen Lösung, eine Vorauswahl relevanter Situationen und einen konzeptionellen Systementwurf beschränkt.

Das Systemkonzept beinhaltet das sogenannte Vehicle Data Intelligence Device, welches in ein Fahrzeug eingebaut und an die Bus-Systeme angeschlossen wird. Es enthält die vollständige Logik der Lösung. Die Abb. 2 zeigt den groben Systementwurf im Kontext des Fahrzeuges. Genauer wird die Systemintegration des Vehicle Data Intelligence Devices dargestellt mit allen Kommu- nikationswegen innerhalb sowie außerhalb des Fahrzeuges.

Die Abb. 2 verdeutlicht, dass das Device der zentrale Knoten des Gesamtsystems ist. Es wird aber als ein Bestandteil des Fahrzeuges integriert. Durch die Bus-Systeme, wie beispielsweise CAN und Flexray wird eine Kommunikation zwischen dem Device und den Sensoren sowie Aktoren sichergestellt. Diese Schnittstellen sind essentiell, da über jene zum einen die Fahrzeugdaten gesammelt und zum anderen Fahrer sowie Fahrzeug konditioniert werden. Die Anbindung erfolgt über gängige Standards der Bus-Systeme.

Gleiches gilt für die Car2X-Kommunikation, die voraussichtlich dem IEEE 802.11 p-Standard entsprechen wird. Neben der Fahrerkonditionierung über die verbauten Aktoren des Fahrzeuges ist eine Einbindung eines Displays angedacht, welches Augmented Reality unterstützt. Dabei ist die Art des Displays nicht festgelegt, um eine maximale Flexibilität sicherzustellen. Es können beispielsweise festverbaute Displays, Einblendungen auf der Frontscheibe oder spezielle Augmented Reality Brillen eingesetzt werden. Eine exemplarische Anwendung für Augmented Reality in Verbindung mit Vehicle Data Intelligence ist die Darstellung von anderen Fahrzeugen hinter Hindernissen, welche die Sicht des Fahrers versperren. Beispielsweise können Fahrzeuge an Kreuzungen hinter Häuserwänden auf dem Display schematisch eingeblendet werden. Somit wird der Fahrer vorkonditioniert und kann entsprechend reagieren. Die Abb. 3 zeigt die benannte Situation.

Zusätzlich ist eine Kamera als Komponente des Systems angedacht, die in Fahrtrichtung filmt und deren Aufnahmen per Bildverarbeitung so ausgewertet werden, dass verwertbare Daten entstehen. Diese Daten werden in das Realtime-Data-Warehouse geladen und für die Erkennung von Mustern bzw. relevanten Situationen genutzt.

Für das Teilen der bekannten Muster und das Aktualisieren der Firmware des Vehicle Data Intelligence Devices wird eine Kommunikation zu einem zentralen Server notwendig. Jenes geschieht zusätzlich zum Austausch der Muster über die Kommunikation der Fahrzeuge untereinander. Das Vehicle Data Intelligence Devise hat die vollständige Logik in sich vereint und der Server dient ausschließlich zum Austausch der ermittelten Daten.

\section{Ausblick}

Die Forschung im Bereich des Data Minings auf Fahrzeugdaten in Echtzeit ist nur gering vorhanden, weshalb ein hohes Potential in der Anwendung existiert. Die frühzeitige Erkennung von Situationen, die ein Eingreifen benötigen, ist ein großes Ziel der Automobilbranche. Durch den Ansatz wird das kooperative Fahren unterstützt, was essentiell für das automatisierte Fahren ist. Je größer die Ausprägung der Automatisierung ist, umso wichtiger ist eine Konditionierung des Fahrzeuges. Ab einer Einteilung der Stufe 2 »teilautomatisiert« nach den 
Automatisierungsgraden der BASt-Projektgruppe (Gasser et al. 2012: 1) ist es zwingend erforderlich, Situationen zu erkennen und das Fahrzeug dementsprechend zu konditionieren. Da die gesamte Branche in diesen Bereich forscht und versucht die Automatisierung voran zu treiben, besteht erhöhter Bedarf an einer solchen Lösung. Dies zeigt u. a. eine Studie der PricewaterhouseCoopers AG aus dem September 2015. Aus der Umfrage geht hervor, dass die für 2016 schon führenden Gebiete Fahrsicherheit und autonomes Fahren (62\% des Gesamtpotentials) bis zum Jahr 2021 stark an Umsatz zulegen werden (PricewaterhouseCoopers 2015).

Der theoretische Grundstein für die Entwicklung von Echtzeit-Data-Mining auf Fahrzeugdaten ist gelegt. So folgen als nächste Schritte die Auswahl der notwendigen Hard- und Software, die Analyse von Methoden zur Validierung von Echtzeitdatenfusion, die Schnittstellendefinitionen sowie die testweise Programmierung von Filtern in der Software-Umgebung Automotive Data and Time-Triggered Framework (ADTF) (Elektrobit Automotive 2015).

\section{Literatur}

Bundesministerium für Bildung und Forschung (2015) Richtlinien zur Förderung von Forschungsinitiativen auf dem Gebiet der „Elektroniksysteme für das vollautomatisierte Fahren (ELEVATE)“ im Rahmen des Förderprogramms "IKT2020“. https://www.bmbf.de/foerderungen/bekanntmachung.php? $\mathrm{B}=1065$. Accessed 18 Feb 2016

Dietmayer K (2014) Autonomous Driving. Universität Ulm. https://www.uni-ulm.de/index.php?id=53479. Accessed 16 Feb 2016

Elektrobit Automotive (2015) EB Assist ADTF. Driver assistance systems start with EB Assist ADTF. https://www.elektrobit. com/products/eb-assist/adtf. Accessed 24 Feb 2016

Fuest B (2015) Ford und Google bauen gemeinsam ein Roboterauto. Die Welt 22 Dec 2015. http://www.welt.de/wirtschaft/article150254592/Ford-und-Google-bauen-gemeinsam-ein-Roboterauto.html. Accessed 15 Feb 2016

Gasser TM, Arzt C, Ayoubi M, Bartels A, Eier J, Flemisch F, Häcker D, Hesse T, Huber W, Lotz C, Maurer M, Ruth-Schumacher S, Schwarz J, Vogt W (2012) Rechtsfolgen zunehmender Fahrzeugautomatisierung. Berichte der Bundesanstalt für Straßenwesen, Unterreihe Fahrzeugsicherheit, F 83. Bundesanstalt für Straßenwesen, Bergisch Gladbach

IEEE Computer Society (2010) IEEE standard for Information technology - Telecommunications and information exchange between systems - Local and metropolitan area networks - Specific requirements. Part 11: Wireless LAN Medium Access Control (MAC) and Physical Layer (PHY) Specifications. Amendment 6: Wireless Access in Vehicular Environments. IEEE Std 802.11 $\mathrm{p}^{\mathrm{TM}}$-2010. Institute of Electrical and Electronics Engineers, New York. ISBN: 978-0-7381$6350-5$

Kubica S (2015) Informationsmanagement im Fahrzeug von Morgen. Forschungskolloquium, Wildau
Nestler Y (2015) Echtzeit für das vernetzte Auto. Funkschau 31 Aug 2015. http://www.funkschau.de/mobile-solutions/artikel/122746/1. Accessed 15 Jan 2016

PricewaterhouseCoopers (2015) Weltweites Marktpotenzial internetbasierter Funktionen in Connected Cars in den Jahren 2016 und 2021. Statista. http://de.statista.com/statistik/daten/studie/324510/umfrage/marktpotential-internetfunktionen-in-pkw. Accessed 12 Feb 2016

\section{Autoren}

Thomas Jacob

Technische Hochschule Wildau

thomas.jacob@th-wildau.de

Stefan Kubica

Technische Hochschule Wildau

stefan.kubica@th-wildau.de

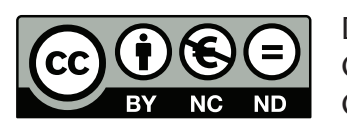

Dieser Beitrag ist unter der Creative-Commons-Lizenz CC BY-NC-ND lizensiert. 